\title{
Identifying Proper Scales on Digital Maps for In-Vehicle Navigation Systems
}

\author{
Anna Wu and Xiaolong Zhang \\ College of Information Science \& Technology, Pennsylvania State University, \\ University Park, PA, 16803, USA \\ \{auw133, lzhang\}@ist.psu.edu
}

\begin{abstract}
Current commercial mobile navigation systems often use a predetermined scale selection schema without considering differences in spatial complexity of locations. To identify what map scales people may need and what spatial features make relevant maps stand out, we conducted an experiment on subjective map selection in a route planning task between two cities in the United States. Our results suggest that the distribution of selected maps is fairly concentrated on those maps that contain spatial information about both the origin and the destination, the current location and the destination, and the transition between different important roads in a route. These results suggest that the choice of map scales should not follow a preset scale rule for diverse locations, and instead, it should be adaptive to the complexity of local roads and decision-making processes.
\end{abstract}

Keywords: Map scale, mobile interface design.

\section{Introduction}

Recently, mobile navigation units have proliferated because of technical advances, increasing effectiveness and reasonable price. A recent report indicates that the global shipments of handsets enabled by the Global Positioning System (GPS) are expected to grow annually at a rate of $26.2 \%$, from 175 million units in 2007 to 560 million units in 2012 [1]. Mobile devices powered by digital maps have become common accessories for automobiles.

The proliferation of GPS devices raises a question of how to better support interaction with digital maps on mobile devices. Compared with paper maps, digital maps are more interactive (e.g. zooming, panning, or even animation) and offer more functions (e.g. destination searching and tool personalizing). However, digital maps on most mobile navigation devices usually look like digitized paper maps. The novelty and complexity of digital maps challenge the approach to apply traditional cartographic theories in digital maps [2]. Issues related to differences in using paper and digital maps have not been much explored. For example, the mobile context asks for special design guidelines to improve the visibility and comprehensiveness of symbols on small screens. Also, while paper maps offer spatial contexts around a focused area, such information is usually not available in the navigation systems that provide automatic 
'turn-by-turn' directions. New theories and empirical studies are needed to improve our understandings of spatial cognition and behaviors with small interactive maps.

This paper focuses on the scale selection of digital maps. One of the advantages of digital maps is that mobile navigation systems can change the scale of displayed digital maps easily. This flexibility, however, raises a question: what scales should be used exactly in guiding wayfinding. A study comparing multiple commercial mobile devices shows that default scale levels for digital maps on these devices are arbitrarily chosen [3]. People need maps at different scales in different tasks. For example, driving in places with different road complexities, such as in the New York City and in the Yellow Stone National Park, should be guided by differently scaled maps. Onesize-fit-all scale design apparently may not work effectively.

In this paper, we study what spatial information people need from maps at different scales. The rest of the paper is organized as follows. Section 2 reviews relevant literature. Section 3 reports our experimental study on map choices in a route planning task. Section 4 discusses the results and implications of our research, and Section 5 concludes the paper with future research directions.

\section{Related Work}

In traditional cartographic field, map schematization, derived from map generalization, is a branch of cartography aiming to simplify information on maps so that when the scale changes, map readers still can get the main meaning with the limited information after selection. The Radical Law [4] is a milestone for that it gives the theoretical answer to how many objects should be represented according to scales and object types. Tufte defined the data density as average date entries on unit graphic area [5]. Since the definition of data entry is vague and difficult in practice, similar to this, the number of objects can also be used for calculating information density [6] so that the system can automatically adjust information to a constant level at different scale levels. Back to 1998, a series of study were carried out on a driving simulator to investigate factors in electronic map-reading, such as number of presented streets, proportion of labeled streets, label font size, text orientation, grid-likeness[7].

While mobile devices greatly expand design space to support real-world tasks and allow people to live in their world with context-aware computers [8], visualization of spatial information for navigational purpose in small displays has caught great attention recently [7, 9-11]. Among many topics in this area, how much information to present is critical in mobile map design: "how much is much"[12]? Now, map schematization is more important for mobile navigation support than traditional paper map or digital maps on desktop because of the limited screen estate and more contextual tasks. Klippel et al. [13] proposed that schematic map should also focus on "cognitively adequacy": the capability to resemble mental knowledge representation and to support cognitive processes. In contrast to spatial information generalization, some maps are designed to increase richness of the representation, e.g. the street view and satellite view of Google Maps [14]. This kind of design aims to reduce the workload in connecting symbolic representation on maps to the real objects in the world, which is one of the main challenges for map-reading. However, research showed that on small devices, more schematic map works better. For example, Dillemuth [11] has compared aerial photos with simplified maps for pedestrians with a handheld 
computer in route-following tasks. Results show that generalized maps get quicker route completion and fewer navigation errors.

Among many factors in map generalizations, scaling is an important one in determining how much information should be presented and in what format. The scale of a map is the ratio of a distance unit on the map to the equivalent distance in the real world. Another factor that influences the information density in a view is level of detail (LOD). LOD can be an attribute fixed to a scale. The selection of map scale also needs to consider legibility that is related to the size and resolution of the display. In the mean time, studies of route planning suggest that spatial knowledge is hierarchically structured and stored based on scales[15] [16]. Tversky notes that people have different mental representation of three spatial knowledge scales: "overview", "view" and "action", and expect different information at each scale[17].

Besides theoretical explanation, some technical attempts have also been proposed by challenging the uniform scale principle. For example, traditional fisheye view is used in city maps [18]. The center of the map view is displayed in normal scale while the marginal part is shrunk. A similar idea called variable-scale maps was proposed in GiMoDig project [19]. The center of variable-scale maps is enlarged to allow better exploration while the marginal part of the map is highly clustered. To solve this problem, radical generalization was proposed to reduce the level of details from center to the edge in radical direction [20].

Despite the critical role in route planning, no practical standard is currently available in mobile map design and few empirical studies reported [3]. What are the selection criteria of map scales for navigational facilitation? To explore this problem, this paper presents a lab experiment to get subjective selections and reasoning in a typical route planning scenario.

\section{Experiment Study}

The purpose of this study is to identify what map scales people may choose in route planning and what features make these maps stand out. The results of this experiment can help us establish a set of design guidelines on what scales maps should be used so that spatial objects that people prefer can be properly displayed in digital maps.

\subsection{Experiment Design}

In order to get the subjective assessment of important scaled maps in route planning tasks, a map selection experiment was conducted. The task was to choose necessary maps among a set of 26 maps in planning a driving route between two residential addresses, Address A and Address B, in two US cities. These two cities are 500-mile apart, and located in two different states. The participants were asked to first plan a route between these two places based on 26 maps, and then to select those maps they would need when executing the route plan.

\subsection{Subject and Apparatus}

Thirty-two college students, 7 females and 25 males, were recruited from a database course. Their ages were between 18 and 28 and with an average of 19.4. They earned one extra credit toward the course because of their participation in the study. 


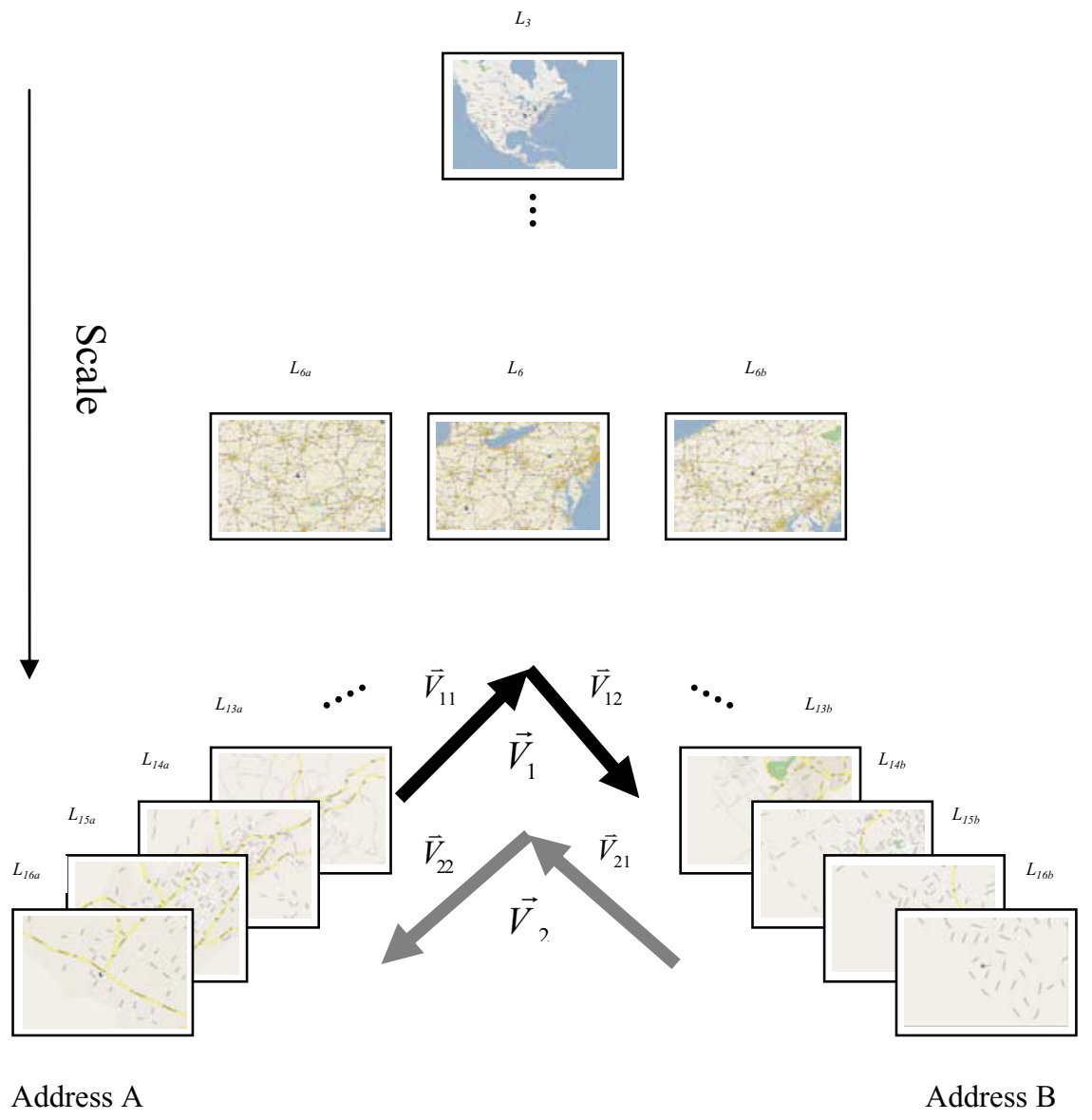

Fig. 1. Maps used in the experiment (Maps from http://maps.google.com)

The materials used in the study were maps from Google Maps. Each map was a color print on $8.5 "$ x 11" (21.59 cm x $27.94 \mathrm{~cm})$ paper. Among 20 available scales in Google Maps from Zoom level 0 to Zoom level 19, we chose 14 scales related to our tasks, from Zoom level 3 to Zoom level 16. The other six scales were either too small or too big. We denoted maps at these scale levels as $L_{3}$ to $L_{16}$ in our study.

Among these maps, small-scale maps from $L_{3}$ to $L_{6}$ include both the origin and the destination There were three maps at Zoom level 6: $L_{6 a}$ had Address A as the center of the map, $L_{6 b}$ had Address B, and $L_{6}$ is centered in the middle of Address A and B. Ten maps, $L_{7 a}$ to $L_{16 a}$, provided detailed information of Address A, and ten maps, $L_{7 b}$ to $L_{16 b}$, were about Address B (Fig. 1.).

From the origin to the destination, the series of selection frequency can be viewed as a vector and each map can be viewed as a dimension. Then two vectors are created:

Address A $\rightarrow$ Address B:

$$
\vec{V}_{1}=\left\{L_{16 a}, L_{15 a}, L_{14 a}, \ldots L_{7 a}, L_{6 a},\left\{L_{6}, \ldots, L_{3}\right\}, L_{6 b}, L_{7 b}, \ldots L_{14 b}, L_{15 b}, L_{16 b}\right\}
$$


Address B $\rightarrow$ Address A:

$$
\vec{V}_{2}=\left\{L_{16 b}, L_{15 b}, L_{14 b}, \ldots L_{7 b}, L_{6 b},\left\{L_{6}, \ldots, L_{3}\right\}, L_{6 a}, L_{7 a}, \ldots L_{14 a}, L_{15 a}, L_{16 a}\right\}
$$

Divided by $L_{5}$, each of this vector can be broke up into two sub-vectors: $\vec{V}_{11}=\left\{L_{16 \mathrm{a}}\right.$, $\left.L_{15 a}, L_{14 a}, \ldots L_{7 a}, L_{6 a}\right\}, \vec{V}_{12}=\left\{L_{6 b}, L_{7 b}, \ldots L_{14 b}, L_{15 b}, L_{16 b}\right\}, \vec{V}_{21}=\left\{L_{16 b}, L_{15 b}, L_{14 b}, \ldots L_{7 b}\right.$, $\left.L_{6 b}\right\}, \vec{V}_{22}=\left\{L_{6 a}, L_{7 a}, \ldots L_{14 a}, L_{15 a}, L_{16 a}\right\}$, and $\vec{V}_{0}=\left\{L_{6}, \ldots, L_{3}\right\}$. Note that $\vec{V}_{0}$ is independent of the traveling direction.

\subsection{Procedure}

Before the test, subjects answered a survey on their usage preferences of GPS systems and maps. A spatial ability test [21] was also conducted before map selection tasks.

In the test, each subject was requested to make two sets of maps. One set to have as minimal maps as possible (Minimal Selection) and the other to have exact 7 maps (7-map Selection). Collecting data about these two sets of maps allows us to know what is the most important spatial information and whether there is a pattern in map selection among people.

To balance the difference that may be caused by the origin and the destination, subjects were divided into two groups with equal number of participants in each. Starting and ending addresses in these two groups were switched.

\subsection{Results}

Spatial Ability Test. We randomly selected our participants with normal spatial ability, which is confirmed by the test of normality of their spatial ability score. ( $\mathrm{p}=$ .200). Correlation test shows no observable correlation between individual's spatial ability and map selection.

Preferences of GPS Usage. Among 32 participants, 14 participants preferred maps and 18 preferred GPS. The reasons for GPS preference include "No risk, feel secure", "Directly give the route, easier", "frustrated in map reading", etc. Example reasons for map preference are "more familiar and freedom", "large view, more conscious of current location", etc.

Selected Maps. Subjects were asked to select two sets of maps in two tasks. One task was to select minimal maps, and it can help us understand what the most important maps are, The other task was to select exact 7 maps, and from this task, we hope to examine whether there is a pattern of map selections among subjects. The median of maps selected is 6 . This number is very close to the number of maps for the second task. Given the minor difference between the results in these two tasks, we analyzed our data from the 7-map selection task.

Familiarity Effect. Most participants were familiar with one location but not the other (Table 1). We want to see whether the familiarity of a location may affect map selection. 
Table 1. Participants' familiarity with two locations

\begin{tabular}{ccc}
\hline Familiarity & Location A & Location B \\
\hline Familiar & $\mathrm{n}=23$ & $\mathrm{n}=0$ \\
Unfamiliar & $\mathrm{n}=9$ & $\mathrm{n}=32$ \\
\hline
\end{tabular}

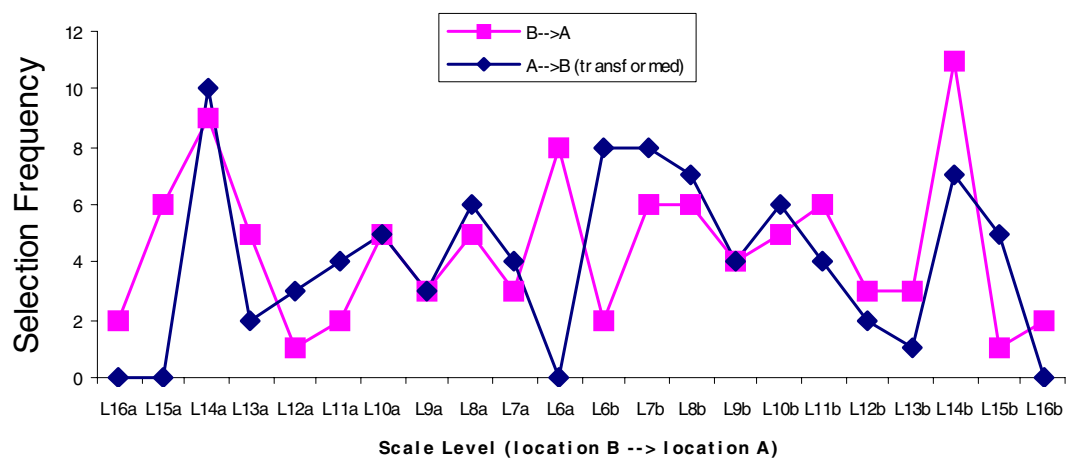

Fig. 2. Comparison of map selection in two groups in 7-map Selection

We compared the map selections between two groups. By excluding those large scale maps that include both the origin and the and start point $\left(\vec{V}_{0}\right)$, we focused on $\vec{V}_{11}$ with $\vec{V}_{21}, \vec{V}_{12}$ with $\vec{V}_{22}$. As shown in Fig. 2., two groups' selections seem to have similar trend, with difference near scale level 6. Comparing the choices of Map $L_{6 a}$, eight counts, and Map $L_{6 b}$, ten counts, we did not find significant difference. Using

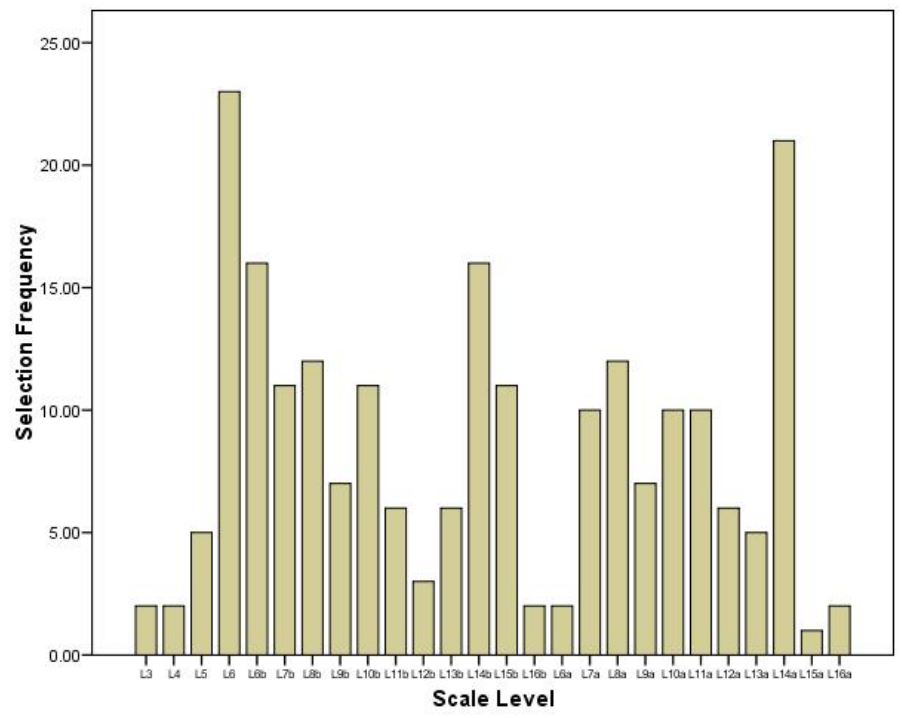

Fig. 3. Selection frequency of maps in different scales 
Table 2. Example reasons for map selection

\begin{tabular}{|c|c|}
\hline Map & Samples of participants' comments \\
\hline$L_{6} \& L_{6 b}$ & $\begin{array}{l}\text { - Good compromise, shows both areas } \\
\text { - } \quad \text { the largest map with both end points marked and a } \\
\text { clear path to each, easy to gauge where you are } \\
\text { based on real-world street signs } \\
\text { - Has all major interstates where most the travel will } \\
\text { take place } \\
\text { - It showed all the routes you could possibly take } \\
\text { - } \text { L }_{6 b} \text { is more readable for carefully examining Loca- } \\
\text { tion B. }\end{array}$ \\
\hline$L_{14 a} \& L_{14 b}$ & $\begin{array}{l}\text { - } \quad \text { This map shows many roads close to my destination } \\
\text { - } \quad \text { Shows the major road the destination is next to } \\
\text { - } \quad \text { Shows how to get from start destination to major } \\
\text { highways }\end{array}$ \\
\hline
\end{tabular}

Distances Similarity Measures for Interval Data (cosine), we found that $\vec{V}_{11}$ is similar to $\vec{V}_{21}$ with a cosine value of .758 , and $\vec{V}_{12}$ is similar to $\vec{V}_{22}$ with a value of 861 . This result suggests that in general, familiarity have no influence over map selection. Thus, in the following analysis, we combine the selection from two groups.

Map Selection. Fig. 3 shows the frequency distribution of selected maps. L6 is the map selected most. This map shows both the origin and the destination at the largest possible scale among all maps. Following it are Map L14a, L14b, and L6b.

Participants provided comments about why these maps were chosen (Table 2). Based on comments by subjects, we summarized the major reasons behind selection of these maps.

Map $L_{6}, L_{6 b}$ : the largest map with both the origin and the destination; providing a good overview of main roads.

Map $L_{14 a}$ and $L_{14 b}$ : showing the transition between highways to local roads; offering detailed about the origin/destination with street names.

\section{Discussion}

The results of our map-selection experiment indicate the selection is fairly concentrated to some particular maps. Highly selected maps include those contains both the origin and the destination, and those with, detailed maps with transitions between local main streets and the destination/origin. Some maps were rarely selected, for example maps at too large or too small scales. Such results suggest that relative spatial relationship between the origin and the destination be a critical concern for users, no matter what scales maps are.

Our results also indicate the importance of an overview of available alternatives to route planning. Subject comments indicate that they need information about "all the 
routes" that are possible to take. This implies that users are not confined to a particular route and may need information about other options.

These results suggest a couple of design guidelines in choosing map scales so that people can obtain critical spatial information:

- Choosing a Map Scale at Which users Can Easily See Where Key DecisionMaking Points are. In our study, the origin and destination are two decisionmaking points. When these two points are far away, always showing the origin and the destination on in a small display is less useful, because this approach will not make sufficient route details visible. However, a route usually includes multiple decision-making points, such as turning intersections and highway entries/exits, and informing users where the next decision point is could be valuable. Thus, a map scale can be calculated based on the distance of two consecutive decisionmaking points. This can be easily achieved in current GPS-based systems, because decision-making points are usually pre-calculated.

- Choosing a Map Scale at Which Users Can Easily See Alternatives between Two Decision-Making Points. This approach involves information about the distance between two points, as well as the size of area that all possible routes may cover. Current navigation systems usually focus on suggesting one route, and do not calculate different routes unless drivers change the prescribed route. Thus, to achieve this goal, systems need to be improved so that they can dynamically determine multiple routes between the current location and the next decision making point.

There are some limitations of our study. First, the task of our study is route planning, and does not include route following, which is more dynamic and requires more diverse spatial information. The difference between these two tasks may lead to different results in map selection. Second, our maps are static, although they are the reproduction of digital maps. Making sense of individual static maps at different scales could be more challenging than reading dynamically updated digital maps. This may affect the decisions of map selection.

\section{Conclusion and Future Work}

In this paper, we reported a study to understand what map features may affect the selection of maps at different scales. Through an experiment, we found that users prefer those maps that allow them to see key decision-making points and alternative routes. These results have implications for multiscale map design on mobile devices by suggesting what scales to use so that users can find what they need. We will extend our work in two directions: including more task scenario types with different travel ranges and information densities under our current approach, and developing a system prototype on mobile devices based on our design guidelines to test it on a driving simulator to see whether our scale selection criteria can work better than current commercial systems. 


\section{References}

1. Berg Insight: GPS-enabled handset shipments to reach 560 million units in 2012, http: / /www. intomobile.com/2008/01/15/berg-insight-gpsenabled-handset-shipments-to-reach-560-million-units-in2012.html

2. Meng, L.: Missing Theories and Methods in Digital Cartography. In: 21st International Cartographic Conference (2003)

3. Dillemuth, J., et al.: Choosing the Scale and Extent of Maps for Navigation with Mobile Computing Systems. Journal of Location Based Services 1(1), 46-61 (2007)

4. Töpfer, F., Pillewizer, W.: The Principles of Selection, A Means of Cartographic Generalization. The Cartographic Journal 3(1), 10-16 (1966)

5. Tufte, E.R., Schmieg, G.M.: The Visual Display of Quantitative Information. American Journal of Physics 53, 1117-1118 (1985)

6. Woodruff, A., et al.: Constant Information Density in Zoomable Interfaces. In: Proceedings of the working conference on Advanced visual interfaces, pp. 57-65. ACM, New York (1998)

7. Brooks, A.: Map Design: a Simulator Evaluation of the Factors Affecting the Time to Read Electronic Navigation Displays. UMTRI-98-7, University of Michigan, Ann Arbor, ITS Research Center of Excellence (1998)

8. Weiser, M.: Hot Topics-Ubiquitous Computing. Computer 26(10), 71-72 (1993)

9. Danado, J., et al.: Mobile Environmental Visualization. Cartographic Journal 42, 61-68 (2005)

10. Edwardes, A., et al.: Portrayal and Generalisation of Point Maps for Mobile Information Services. In: Meng, L., Zipf, A., Reichenbacher, T. (eds.) Map-based Mobile Services: Theories, Methods and Implementations (2005)

11. Dillemuth, J.: Map Design Evaluation for Mobile Display. Cartography and Geographic Information Science 32, 285-301 (2005)

12. Meilinger, T., et al.: How Much Information Do You Need? Schematic Maps in Wayfinding and Self Localisation. In: Barkowsky, T., Knauff, M., Ligozat, G., Montello, D.R. (eds.) Spatial Cognition 2007. LNCS, vol. 4387, pp. 381-400. Springer, Heidelberg (2007)

13. Klippel, A., et al.: The Cognitive Reality of Schematic Maps. In: Meng, L., Zipf, A., Reichenbacher, T. (eds.) Map-Based Mobile Services: Theories, Methods and Implementations. Springer, Berlin (2005)

14. Google Maps, http://maps.google.com/

15. Wiener, J.M., Mallot, H.A.: 'Fine-to-Coarse' Route Planning and Navigation in Regionalized Environments. Spatial Cognition \& Computation 3(4), 331-358 (2003)

16. Chown, E., et al.: Prototypes, Location, and Associative Networks (PLAN): Towards a Unified Theory of Cognitive Mapping. Cognitive Science 19(1), 1-51 (1995)

17. Tverksy, B.: Levels and structure of spatial knowledge. In: Kitchin, R., Freundschuh, S. (eds.) Cognitive Mapping: Past, Present and Future. Routledge, London (2000)

18. Rauschenbach, U.: The Rectangular Fisheye View as an Efficient Method for the Transmission and Display of Large Images. In: Proceedings of International Conference on Image Processing, pp. 115-119. IEEE, Los Alamitos (1999)

19. Harrie, L., et al.: A Variable-Scale Map for Small-Display Cartography. International Archives of Photogrammetry Remote Sensing and Spatial Information Sciences 34(4), 237 242 (2002)

20. Rappo, A.: Fischaugenprojektionen mit distanzabhängiger Informationsdichte für die kartographische Visualisierung auf kleinen Bildschirmen. Universität Zürich, Cited from Reichenbacher, T. (2004). Mobile Cartography -Adaptive Visualisation of Geographic Information on Mobile Devices. Doctoral Thesis, Technical University of Munich (2003)

21. Ekstrom, R.B., et al.: Kit of Reference Tests for Cognitive Factors. Educational Testing Services, Princeton (1963) 